\title{
A novel way of determining gestational age upon the birth of a child
}

\section{Kumanan Wilson ${ }^{1,2,3}$, Victoria Ward ${ }^{4}$, Pranesh Chakraborty ${ }^{5,6}$, Gary L Darmstadt ${ }^{4}$}

${ }^{1}$ Department of Medicine, University of Ottawa, Ottawa, Ontario, Canada

${ }^{2}$ Clinical Epidemiology Program, Ottawa Hospital Research Institute, Ottawa, Ontario, Canada

${ }^{3}$ Bruyère and Hospital Research Institutes, Ottawa Ontario, Canada

${ }^{4}$ Department of Pediatrics, Stanford University School of Medicine, Stanford, California, USA

${ }^{5}$ Department of Pediatrics, Children's Hospital of Eastern Ontario and University of Ottawa, Ottawa, Ontario, Canada

${ }^{6}$ Newborn Screening Ontario, Ottawa, Ontario, Canada

$\mathrm{P}$ reterm birth is the leading cause of infant morbidity and mortality globally [1]. Determining whether an infant was born preterm can be challenging, especially in low-resource settings due to a paucity of prenatal care and dating ultrasounds, the unreliability of recall of last menstrual period, and recognised limitations of Ballard score and other newborn clinical assessment and anthropometric measurements [2]. Accurate estimation of gestational age (GA) is important in informing the medical care of the newborn and accurately assessing neurocognitive development. GA dating is also necessary for population-level determination of preterm birth rates as well as appropriate resource and intervention allocations. There is a need for better data that provide robust estimates of the burden of preterm birth in low-resource settings.

\section{OPPORTUNITIES FROM METABOLIC GA TESTING}

The accurate estimation of gestational age is critical in informing resource allocation and care for infants born too early or too small, yet this can be challenging in resource poor setting where gestational age estimates are often based on less accurate methods such as last menstrual period or clinical or anthropometric assessments.
Recently, novel methods for establishing an infant's GA have emerged that may be able to overcome some of the existing limitations. One such approach involves using an established public health procedure, a heel prick blood spot typically used for newborn screening [3]. Newborn screening is a routine practice in many high-income countries, wherein a blood spot is collected via heel prick from newborns to screen them for a variety of rare, treatable conditions. The blood spot is analysed to determine levels of several analytes including acylcarnitines, amino acids, and endocrine markers. Abnormalities in specific analytes or ratios of analytes may suggest an underlying disorder. Infants who are identified as "screen positive" for one or more of the screened conditions undergo confirmatory testing and, ideally, receive prompt, definitive treatment. 
Levels of the analytes measured by newborn screening vary by the GA of an infant at birth [4]. While this can impact the performance of newborn screening, it has also created an opportunity to use the levels of these analytes at birth to determine the infant's GA. Three separate groups in Canada and the United States have successfully created algorithms that use birthweight and analytes from their newborn screening programmes to accurately estimate GA within one to two weeks [4-6]. The addition of fetal-to-adult hemoglobin ratio, measured when screening for hemoglobinopathies, has further improved the predictive ability of these algorithms [7]. Preliminary data from studies seeking to validate these algorithms in low-resource settings have shown promising results and suggest potential utility for these models on cord blood samples in addition to samples derived from heel prick.

Further validation of this approach is necessary to determine to what extent local factors may affect metabolic profiles, which in turn could impact the accuracy and generalisability of metabolic-derived estimates of GA. We are currently in the process of implementing and further evaluating this approach to GA estimation in multiple low-resource settings in sub-Saharan Africa and South Asia, including Kenya, Zambia, Zim-

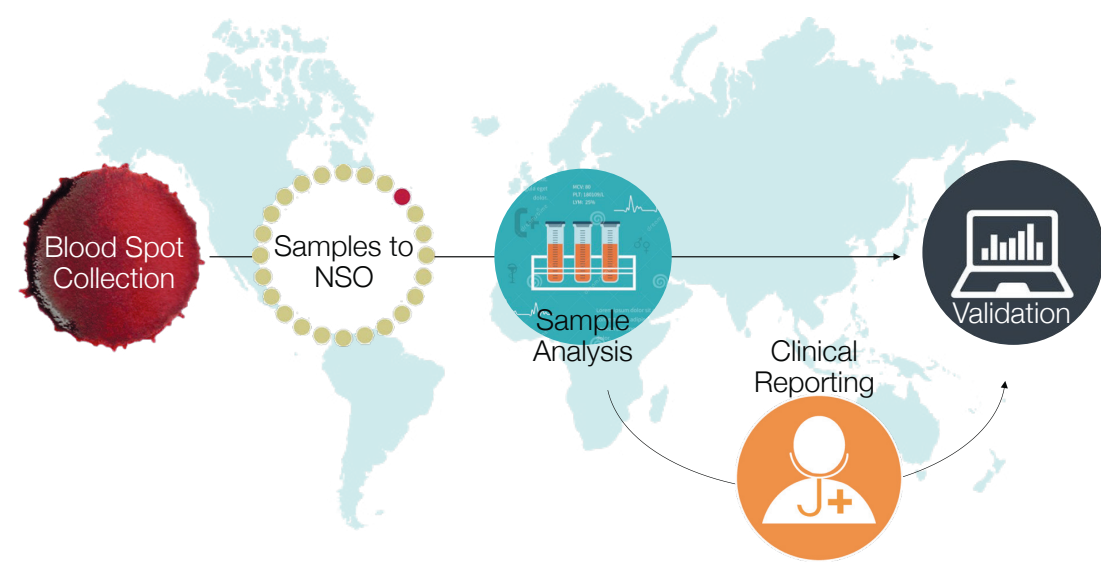

Figure 1. Gestational age assessment in international preterm birth cohorts. babwe, and Bangladesh [8]. Heel prick and/or cord blood samples are being obtained from infants in these sites and sent to Newborn Screening Ontario laboratories for further analysis. This approach will generate population-level estimates of rates of preterm birth and small-forgestational age (SGA) infants, ultimately intended to inform global and regional estimates of the burden of preterm birth. Our approach also includes screening for select metabolic disorders which can be successfully followed up and treated at the sites [9] (Figure 1). The University of California - San Francisco has completed a similar study in Uganda [10].

\section{CHALLENGES TO THIS APPROACH}

Logistical challenges with obtaining samples within an appropriate timeframe (ideally 24-72 hours after birth) and then shipping them to an external laboratory under conditions that preserve the quality of the blood spots are important to address. Ultimately, the goal will be to develop in-country capacity and point-of-care diagnostics that will permit these analyses at the time of birth to inform care for individual infants. There are also cultural concerns to be understood and addressed that may affect willingness of families to provide either heel prick or cord blood samples. Another challenge is the adaptation of algorithms developed using blood spot data to estimate population rates of SGA. Existing algorithms can be enhanced using machine learning approaches to improve model performance, and could be further reiterated for SGA-specific estimations [11].

\begin{abstract}
The novel use of metabolic gestational age algorithms based on newborn screening data offers an opportunity to improve postnatal gestational age estimates in low resource settings, identify treatable conditions, determine risk for specific health conditions, and estimate population-level rates of preterm birth to guide health system planning and resource allocation for improved cost-effectiveness and impact of maternal and child health policies and programmes.
\end{abstract}

Is this approach worth the cost? According to our estimates, the incremental cost per preterm infant correctly classified by the metabolic algorithm is US\$11 542, and per SGA infant is US\$688 [12]. Determining whether these costs are acceptable in resource poor settings is an important consideration. While birth weight alone is reasonable at identifying appropriate-for-gestational age (AGA) preterm infants, our analyses suggest that the real value of this approach may be to help identify SGA infants and distinguish them from preterm infants. Ultimately, the value of this approach is dependent on the reduction in morbidity, mortality and health resource utilisation that this identification can realise.

To optimise benefit for costs, additional potential value to the collection of these samples should be evaluated. An immediate obvious benefit is the potential to guide the care of appropriately recognised preterm infants, provided that metabolic data can be analysed and processed promptly. Moreover, the analysis of the samples can provide newborn screening value by identifying infants at risk of treatable conditions such as congenital hypothyroidism and hemoglobinopathies. 


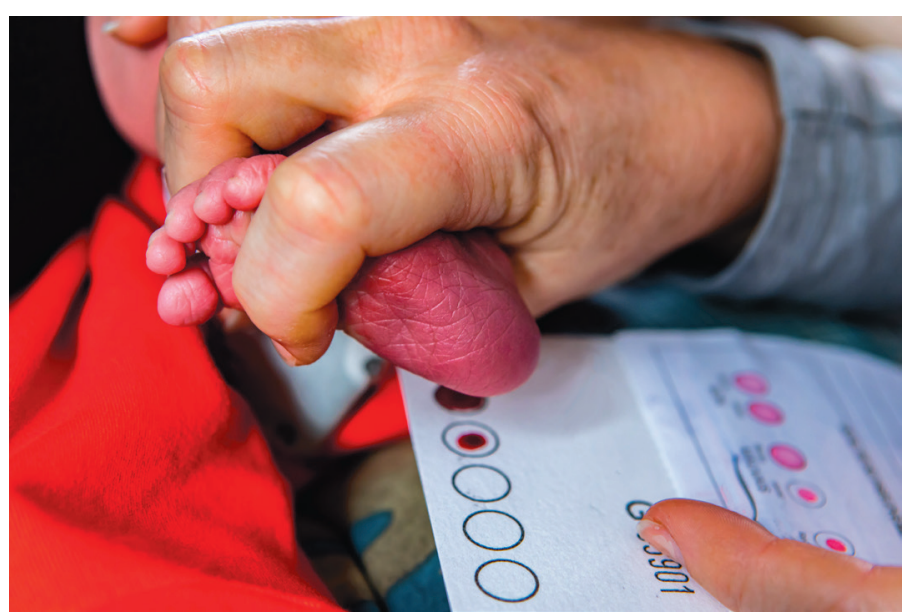

Photo: Close up details as a family doctor carries out a heel prick blood test during newborn baby health screening, squeezing drops from foot for analysis (Valmedia via: https://www.shutterstock.com/image-photo/close-details-family-doctor-carriesout-1628211025).
Looking to the future, work is under way to determine whether there is additional value to the analysis of blood spots, including their predictive value for a host of conditions including early neonatal mortality and neonatal sepsis $[13,14]$. Blood spots can also potentially be used for genetic analyses. Expanded metabolomic panels that go beyond the analytes collected for newborn screening purposes may also improve the predictive value of the blood spots for identifying modifiable risk factors and treatable conditions. In addition, the predictive and diagnostic utility of newborn blood spots may be expanded through integration of data derived from maternal blood and maternal health history [15].

\section{CONCLUSION}

We envision a future where a heel prick at birth could inform caregivers of the GA of a child, distinguish SGA from preterm infants, identify treatable conditions and determine risk for specific health conditions. This data could also provide population-level estimates of preterm birth to guide health system planning and resource allocation for improved cost-effectiveness and impact of maternal and child health policies and programmes.

\footnotetext{
Ethics approval: The ongoing research referred to in this viewpoint was approved by the Ottawa Health Sciences Net-
work Research Ethics Board (20180330-01H), Children's Hospital of Eastern Ontario Research Ethics Board (18/58X),
the Stanford University School of Medicine Institutional Review Board (44656), the Kenya Medical Research Institute Sci-
entific and Ethics Review Unit (SSC 2880), the University of Zambia Biomedical Research Ethics Committee(015-06-17),
the Bangladesh Institute of Child Health (BICH-ERC-01-01-2019), and the Research Council of Zimbabwe (03744).
Funding: The work described in this perspective paper is funded by the Bill \& Melinda Gates Foundation [OPP1184574
(KW) and (OPP1182996 (GLD)].
Authorship contributions: KW conceptualized and is the guarantor of the manuscript, and both he and GLD wrote
the manuscript and conducted the literature search. All authors approved the final manuscript as submitted and agree
to be accountable for all aspects of the work.
Competing interests: The authors have completed the ICMJE Disclosure of Interest Form (available upon request from
the corresponding author), and declare no conflicts of interest.
}

1 March of Dimes, PMNCH, Save the Children, WHO. Born Too Soon: The Global Action Report on Preterm Birth. Geneva, Switzerland; 2012

2 Lee AC, Mullany LC, Ladhani K, Uddin J, Mitra D, Ahmed P, et al. Validity of Newborn Clinical Assessment to Determine Gestational Age in Bangladesh. Pediatrics. 2016;138:e20153303. Medline:27313070 doi:10.1542/peds.2015-3303

3 Mundel T. Innovation: How a 50-Year-Old Drop of Blood Helps Solve an Urgent Global Health Challenge I Impatient Optimists. Impatient Optimists. 2017.

4 Wilson K, Hawken S, Potter BK, Chakraborty P, Walker M, Ducharme R, et al. Accurate prediction of gestational age using newborn screening analyte data. Am J Obstet Gynecol. 2016;214:513.el-9. Medline:26519781 doi:10.1016/j.ajog.2015.10.017

5 Jelliffe-Pawlowski LL, Norton ME, Baer RJ, Santos N, Rutherford GW. Gestational dating by metabolic profile at birth: A California cohort study. Am J Obstet Gynecol. 2016;214:511.e1-13. Medline:26688490 doi:10.1016/j.ajog.2015.11.029

6 Ryckman KK, Berberich SL, Dagle JM. Predicting gestational age using neonatal metabolic markers. Am J Obstet Gynecol. 2016;214:515.e1-13. Medline:26645954 doi:10.1016/j.ajog.2015.11.028

7 Wilson K, Hawken S, Murphy MSQ, Atkinson KM, Potter BK, Sprague A, et al. Postnatal Prediction of Gestational Age Using Newborn Fetal Hemoglobin Levels. EBioMedicine. 2017;15:203-9. Medline:27939425 doi:10.1016/j.ebiom.2016.11.032

8 Bota AB, Ward V, Hawken S, Wilson LA, Lamoureux M, Ducharme R, et al. Metabolic gestational age assessment in low resource settings: a validation protocol. Gates Open Res. 2021;4:150. Medline:33501414 doi:10.12688/gatesopenres.13155.2

9 Murphy MSQ, Chakraborty P, Pervin J, Rahman A, Wilson LA, Lamoureux M, et al. Incidental screen positive findings in a prospective cohort study in Matlab, Bangladesh: insights into expanded newborn screening for low-resource settings. Orphanet J Rare Dis. 2019;14:25. Medline:30700313 doi:10.1186/s13023-018-0993-1 
10 Oltman SP, Jasper EA, Kajubi R, Ochieng T, Kakuru A, Adrama H, et al. Gestational age dating using newborn metabolic screening: A validation study in Busia, Uganda. J Glob Health. 2021;11:04012. Medline:33692896 doi:10.7189/jogh.11.04012

11 Hawken S, Ducharme R, Murphy MS, Olibris B, Brianne Bota A, Wilson LA, et al. Development and external validation of machine learning algorithms for postnatal gestational age estimation using clinical data and metabolomic markers Authors and affiliations. medRxiv. 2020. 2020.07.21.20158196.

12 Coyle K, Quan AML, Wilson LA, Hawken S, Bota AB, Coyle D, et al. Cost-effectiveness of a gestational age metabolic algorithm for preterm and small-for-gestational-age classification. Am J Obstet Gynecol MFM. 2021;3:100279. Medline:33451597 doi:10.1016/j.ajogmf.2020.100279

13 Fell DB, Hawken S, Wong CA, Wilson LA, Murphy MSQ, Chakraborty P, et al. Using newborn screening analytes to identify cases of neonatal sepsis. Sci Rep. 2017;7:18020. Medline:29269842 doi:10.1038/s41598-017-18371-1

14 Oltman SP, Rogers EE, Baer RJ, Anderson JG, Steurer MA, Pantell MS, et al. Initial Metabolic Profiles Are Associated with 7-Day Survival among Infants Born at 22-25 Weeks of Gestation. J Pediatr. 2018;198:194-200.e3. Medline:29661562 doi:10.1016/j. jpeds.2018.03.032

15 Stelzer IA, Ghaemi MS, Han X, Ando K, Hédou JJ, Feyaerts D, et al. Integrated trajectories of the maternal metabolome, proteome, and immunome predict labor onset. Sci Transl Med. 2021;13:eabd9898. Medline:33952678 doi:10.1126/scitranslmed.abd9898

\section{Correspondence to:}

Kumanan Wilson

Ottawa Hospital Research Institute

Civic Campus

1053 Carling Ave

Box 684

Ottawa ON, K1Y 4E9

Canada

kwilson@ohri.ca 